\title{
Política de autogestión hospitalaria en Chile: percepciones de los tomadores de decisiones
}

\author{
Claudio A. Méndez, ${ }^{1}$ Christian Miranda, ${ }^{2}$ \\ M. Cristina Torres ${ }^{1}$ y Myriam Márquez ${ }^{3}$
}

Forma de citar Méndez CA, Miranda C, Torres MC, Márquez M. Política de autogestión hospitalaria en Chile: percepciones de los tomadores de decisiones. Rev Panam Salud Publica. 2013;33(1):47-53.

RESUMEN Objetivo. Conocer las percepciones de los tomadores de decisiones respecto de la etapa de implementación de la politica de autogestión hospitalaria en dos hospitales de alta complejidad del sur de Chile.

Métodos. Se realizó un estudio cualitativo descriptivo y exploratorio basado en entrevistas semiestructuradas en profundidad a tomadores de decisiones de los hospitales Regional de la ciudad de Valdivia y San José de la ciudad de Osorno, durante el período de agosto de 2010 a diciembre de 2011. Se seleccionó una muestra por conveniencia de 26 tomadores de decisiones. Las 26 entrevistas fueron grabadas y transcritas en forma literal. El análisis de la información se hizo utilizando la técnica de análisis de contenido, en su aproximación inductiva.

Resultados. Para los entrevistados, la conceptualización de la autogestión está determinada por la autonomía para la toma de decisiones respecto de la asignación de recursos y el financiamiento de la provisión de servicios de salud en las instituciones hospitalarias. También manifestaron que para mejorar la etapa de implementación se deben incluir políticas de recursos humanos y de financiamiento de la función de provisión de servicios de salud. A las debilidades, por su parte, las relacionaron con la ausencia de capacidades organizacionales y competencias gerenciales de los equipos de salud para la implementación de los cambios.

Conclusiones. La política de autogestión hospitalaria es conceptualizada desde la autonomía financiera, y su implementación está determinada por las brechas de capacidad que persisten en el diseño de la política.

Palabras clave Política de salud; administración hospitalaria; servicios de salud; investigación sobre servicios de salud; Chile; América Latina.

El desempeño de los hospitales ha sido parte permanente de la agenda de reformas sectoriales. Principalmente a través de los procesos de descentraliza-

\footnotetext{
1 Instituto de Salud Pública, Facultad de Medicina, Universidad Austral de Chile. La correspondencia se debe dirigir a Claudio A. Méndez. Correo electrónico: claudiomendez@uach.cl

2 Departamento de Educación, Facultad de Ciencias Sociales, Universidad de Chile.

3 Instituto de Salud Sexual y Reproductiva, Facultad de Medicina, Universidad Austral de Chile.
}

ción introducidos por las reformas a los sistemas de salud, países de América Latina, Asia y Europa han diseñado e implementado políticas dirigidas a ampliar la autonomía de los hospitales, con el propósito de mejorar la eficiencia y la calidad de los servicios de salud (1-9).

En Chile, la reforma sanitaria introdujo la priorización de intervenciones garantizadas bajo un enfoque de derechos. Actualmente, 69 problemas e intervenciones de salud se encuentran bajo el régimen de garantías explícitas en salud (GES), las cuales aseguran acceso, oportunidad de atención, protección financiera y calidad. A nivel organizacional, uno de sus principales cambios involucró la conversión de los hospitales de alta complejidad del país en establecimientos de autogestión en red (EAR) (10). Un hospital de alta complejidad es aquel con el más alto nivel de servicios 
de apoyo diagnóstico y terapéutico, disponibilidad y grado de especialización de los recursos humanos, equipamiento y capacidad de resolución (11).

Previo a la reforma, los hospitales públicos no contaban con autonomía para la gestión de sus recursos por parte de los equipos directivos (12). Así, la política de autogestión buscó incorporar un nuevo modelo de gestión pública en los hospitales, basado en la creación de espacios de mayor flexibilidad y autonomía para la asignación de los recursos y la gestión de su presupuesto (13). Asimismo, procuró modelar el desarrollo de la provisión pública de servicios de salud en una lógica de redes integradas, alinear a los hospitales con el proceso de cambio de modelo de atención y generar las condiciones organizacionales para ajustar la oferta de provisión de servicios a las nuevas necesidades de salud introducidas por las GES (14).

A los hospitales de alta complejidad, la acreditación como EAR les confería un status que supone mayor eficiencia en la gestión, tercerizar servicios de mayor costo, generar carteras de proyectos de desarrollo y celebrar convenios de colaboración con otras instituciones de la red (15). Para ser acreditados como EAR, los hospitales debían avanzar en la implementación de un plan de desarrollo estratégico que considerara elementos relacionados con la política y un plan de acción de recursos humanos, de gestión financiera, de satisfacción de los usuarios, de gestión clínica y de planificación y control de gestión (14). No obstante, al año 2008 apenas 11 de los 59 hospitales de alta complejidad habían completado el proceso que los acreditaba como EAR (16).

Si bien en la actualidad los 59 hospitales incluidos en la ley de Autoridad Sanitaria y Gestión se encuentran adscritos al régimen de EAR por el solo imperio de la ley, no existe evidencia que describa cómo los tomadores de decisiones de los hospitales perciben la implementación de dicha política.

En Chile, son los profesionales directivos con influencia formal en la toma de decisiones los principales involucrados en liderar los equipos de salud y asumir las responsabilidades por los resultados obtenidos de las políticas del sector. Tales responsabilidades subrayan la relevancia de sus percepciones respecto de las dificultades, fortalezas y brechas que aún persisten en los hospitales respecto del diseño e implementación de la polí- tica de autogestión. El presente trabajo tiene por objetivo justamente conocer las percepciones de los tomadores de decisiones respecto de la etapa de implementación de la política de autogestión hospitalaria en dos hospitales de alta complejidad del sur de Chile.

\section{MATERIALES Y MÉTODOS}

Se condujo un estudio cualitativo, descriptivo y exploratorio. Este tipo de diseño es adecuado cuando se busca indagar y describir un fenómeno de interés poco comprendido en el momento, con la idea de generar nuevos presupuestos de investigación y unidades de análisis (17). Dada la falta de evidencias respecto de la implementación de la política de autogestión hospitalaria en red, se consideró que este diseño podría ser el más apropiado para alcanzar el objetivo de la investigación.

Los dos hospitales seleccionados corresponden a centros de alta complejidad situados al sur del país, y tienen 524 y 337 camas disponibles para la atención de pacientes en la ciudad de Valdivia, Región de Los Ríos, y en la ciudad de Osorno, provincia homónima, respectivamente. La Región de los Ríos cuenta con unos 350000 habitantes y la provincia de Osorno, administrativamente dependiente de la Región de Los Lagos, unos 220 000. La selección de estos hospitales obedeció a que ambos presentaron evaluaciones contrapuestas respecto de su avance para acreditar como EAR. El Hospital Regional de Valdivia (HRV) registró puntuaciones altas en las mediciones de los años 2008 y 2009, comparadas con un bajo desempeño del Hospital San José de Osorno (HSJO) (18). Esta diferencia de puntaciones permitió contrastar las percepciones de los tomadores de decisiones de ambos establecimientos, los cuales, aun cuando formalmente son considerados EAR, se encuentran en distintas fases de implementación para la provisión de servicios a dos poblaciones con perfiles epidemiológicos similares.

Se seleccionó una muestra por conveniencia de profesionales con influencia formal en el proceso de toma de decisiones (19). Por su relevancia en la implementación de cambios y liderazgo en los equipos de salud, se definió como profesional con influencia formal en la toma de decisiones a aquellos que ocupaban cargos de responsabilidad directiva: directores(as), subdirectores(as) y jefes(as) de servicios clínicos. Al mismo tiempo, para estar seguros de que los profesionales habían participado en la etapa de implementación, fueron considerados aquellos que al momento de realizarse el estudio tenían una relación contractual de al menos dos años con la institución.

La muestra fue integrada por 26 profesionales, quienes fueron contactados en forma personal por uno de los investigadores. Asimismo, se solicitó autorización a las direcciones administrativas y la aprobación del protocolo de investigación por los comités de ética de los servicios de salud de Valdivia y de Osorno. Todos los profesionales contactados aceptaron participar en el estudio.

El trabajo de campo se realizó desde agosto de 2010 a diciembre de 2011. Para la recolección de la información se utilizó la técnica cualitativa de entrevista individual semiestructurada en profundidad (20), a través de la cual se abordaron los cambios provenientes de la política introducidos en ambos hospitales (cuadro 1). Se buscó la saturación global de las dimensiones exploradas a través de las percepciones. Las entrevistas duraron entre 20 minutos y 1 hora, y se realizaron en dependencias de los hospitales de adscripción de los entrevistados. Las entrevistas fueron ad-

\section{CUADRO 1. Entrevista semiestructurada aplicada a los tomadores de decisiones de los hospitales Regional de Valdivia y San José de Osorno, Chile, agosto de 2010 a diciembre de 2011}

Preguntas

\footnotetext{
- ¿Qué entiende usted por autogestión?

- ¿Cuáles cree usted son los propósitos de autogestionar los hospitales?

- ¿Cuáles cree usted son las consecuencias para la gestión hospitalaria?

- ¿Cuáles han sido los obstáculos organizacionales para implementar la autogestión?

- ¿Cuáles cree usted han sido las dificultades de los equipos para la implementación?

- ¿Cuáles han sido las fortalezas organizacionales para la implementación?

- ¿Cuáles han sido las fortalezas de los equipos para la implementación?

- ¿Qué cambios cree usted son necesarios para mejorar la autogestión?
} 
CUADRO 2. Unidades de análisis y esquemas de codificación aplicados a los discursos de los tomadores de decisiones de los hospitales Regional de Valdivia y San José de Osorno, agosto de 2010 a diciembre de 2011

\begin{tabular}{ll}
\hline \multicolumn{1}{c}{ Unidad de análisis } & \multicolumn{1}{c}{ Código } \\
\hline Autogestión & - Concepto \\
& - Diseño \\
& - Implementación \\
& - Evaluación \\
& - Garantías \\
Provisión & - Gestión \\
& - Modelo \\
& - Integración \\
Recursos humanos & - Retribución \\
& - Desempeño \\
Políticas & - Competencias \\
& - Recursonciamiento humanos \\
& - Integración \\
\hline
\end{tabular}

ministradas solamente por dos personas, con el fin de obtener mayor consistencia y reducir la variación en el enfoque de los temas presentados a los sujetos (21). Las entrevistas fueron grabadas, previa suscripción del documento de consentimiento informado, y transcritas en forma literal. Posteriormente - como criterio de rigor científico - fueron enviadas a los entrevistados para darles la oportunidad de hacer comentarios o correcciones.

Las transcripciones fueron leídas en forma reiterada por el grupo de investigadores y codificadas de acuerdo a la técnica de análisis del contenido en su aproximación inductiva $(22,23)$. Para la reducción de la información, se definieron unidades de análisis y esquemas de codificación (cuadro 2). Posteriormente, se seleccionaron citas literales de las entrevistas, codificadas de acuerdo al hospital de adscripción de los tomadores de decisiones: HRV o HSJO.

La calidad de la investigación se cauteló a través de los criterios de rigor científico de credibilidad, dependencia, confirmabilidad y transferibilidad (2426): la credibilidad mediante la triangulación de investigadores y el envío de las transcripciones a los entrevistados; la dependencia y confirmabilidad, en base a la descripción sistemática de la metodología utilizada y la reflexividad en la conducción del análisis; y la transferibilidad, sustentándola en la homogeneidad estructural y organizacional de la provisión pública del sistema de salud chileno, que permite transferir parte de los hallazgos a otros hospitales de alta complejidad que respondan a las diferentes características de los hospitales estudiados.

\section{RESULTADOS}

De los 26 tomadores de decisiones entrevistados, $76,9 \%$ fueron profesionales del sexo femenino, en tanto que $34,6 \%$ se desempeñaron como médicos cirujanos (cuadro 3). En el cuadro 4 se pueden ver las categorías y subcategorías temáticas que emergieron del análisis de la información.

\section{Categoría de conceptualización y diseño}

En el análisis de la subcategoría de significado, se pudo constatar que los tomadores de decisiones construyen la significancia de la política de autogestión desde la autonomía para la toma de decisiones, para la asignación de recursos financieros y para el establecimiento de normas y protocolos clínicos sobre la provisión de servicios de salud.

[HSJO-01] “. . . significa que el hospital debe ser capaz de sustentarse económicamente, porque antiguamente recibiamos todo de afuera y ahora tenemos - por medio de todos los centros de costos - que ingresar una cantidad de dinero necesaria para poder llevar adelante el hospital. .."

[HRV-13] ". . .consiste básicamente en entregarle una serie de atribuciones por ley a los hospitales, desde el punto de vista de la gestión financiera, de la gestión administrativa, de la gestión de recursos humanos. .."

Por otra parte, en la subcategoría de objetivos de la política, los entrevistados los relacionaron con el establecimiento de procesos dirigidos a fortalecer la eficiencia administrativa y financiera de los hospitales, y aun a responder mejor a las demandas en la provisión de servicios de salud.

[HSJO-02] ". . .en el caso de un hospital, se trata de realizar todas las medidas administrativas necesarias para lograr eficiencia en el manejo de los recursos, con el objeto de cumplir las metas propuestas por el Ministerio y los compromisos de gestión adquiridos..."

[HRV-09] “. . .mejorar la eficiencia y la productividad, disminuir las listas de espera, y otorgar una mejor calidad de atención a los usuarios en forma más oportuna y eficiente. .."

CUADRO 3. Características sociodemográficas y profesionales de una muestra de tomadores de decisiones de los hospitales Regional de Valdivia y San José de Osorno, agosto de 2010 a diciembre de 2011

\begin{tabular}{|c|c|c|c|c|c|c|}
\hline \multirow[b]{3}{*}{ Característica } & \multicolumn{4}{|c|}{ Hospital } & & \\
\hline & \multicolumn{2}{|c|}{$\begin{array}{l}\text { Regional de } \\
\text { Valdivia }\end{array}$} & \multicolumn{2}{|c|}{$\begin{array}{l}\text { San José } \\
\text { de Osorno }\end{array}$} & \multicolumn{2}{|c|}{ Total } \\
\hline & No. & $\%$ & No. & $\%$ & No. & $\%$ \\
\hline \multicolumn{7}{|l|}{ Sexo } \\
\hline Hombre & 1 & 7,1 & 5 & 41,7 & 6 & 23,1 \\
\hline Mujer & 13 & 92,9 & 7 & 58,3 & 20 & 76,9 \\
\hline \multicolumn{7}{|l|}{ Edad (años) } \\
\hline $30-40$ & 3 & 21,4 & 2 & 16,6 & 5 & 19,2 \\
\hline $41-50$ & 3 & 21,4 & 1 & 8,3 & 4 & 15,4 \\
\hline $51-60$ & 7 & 50,0 & 8 & 66,7 & 15 & 57,7 \\
\hline $61-70$ & 1 & 7,1 & 1 & 8,3 & 2 & 7,6 \\
\hline \multicolumn{7}{|l|}{ Profesión } \\
\hline Médico cirujano & 1 & 7,1 & 8 & 66,7 & 9 & 34,6 \\
\hline Enfermera & 9 & 64,3 & 1 & 8,3 & 10 & 38,5 \\
\hline Nutricionista & 0 & 0,0 & 1 & 8,3 & 1 & 3,8 \\
\hline Matrona & 2 & 14,3 & 0 & 0,0 & 2 & 7,7 \\
\hline Químico farmacéutico & 0 & 0,0 & 1 & 8,3 & 1 & 3,8 \\
\hline Administrador público & 1 & 7,1 & 0 & 0,0 & 1 & 3,8 \\
\hline Tecnólogo médico & 0 & 0,0 & 1 & 8,3 & 1 & 3,8 \\
\hline Ingeniero comercial & 1 & 7,1 & 0 & 0,0 & 1 & 3,8 \\
\hline \multicolumn{7}{|l|}{ Años de servicio } \\
\hline $01-10$ & 4 & 28,5 & 5 & 41,7 & 9 & 34,6 \\
\hline $11-20$ & 3 & 21,4 & 1 & 8,3 & 4 & 15,4 \\
\hline $21-30$ & 3 & 21,4 & 3 & 25,0 & 6 & 23,1 \\
\hline $31-40$ & 4 & 28,5 & 3 & 25,0 & 7 & 26,9 \\
\hline Total & 14 & 100,0 & 12 & 100,0 & 26 & 100,0 \\
\hline
\end{tabular}


CUADRO 4. Categorías y subcategorías temáticas emergentes de los discursos de los tomadores de decisiones de los hospitales Regional de Valdivia y San José de Osorno, agosto de 2010 a diciembre de 2011

\begin{tabular}{ll}
\hline \multicolumn{1}{c}{ Categoría } & Subcategoría \\
\hline Conceptualización y diseño & - Significado \\
& - Objetivos \\
& - Diseño \\
Implementación de los & - Cambios \\
cambios organizacionales & - Fortalezas \\
& - Obstáculos \\
Políticas y estrategias & - Equipos de salud \\
& - Financiamiento \\
\hline
\end{tabular}

En la subcategoría de diseño de la política, los tomadores de decisiones identificaron como sus ejes la implementación de mejores sistemas de control financiero para la contención de costos y de procesos administrativos para optimizar el desempeño de los hospitales.

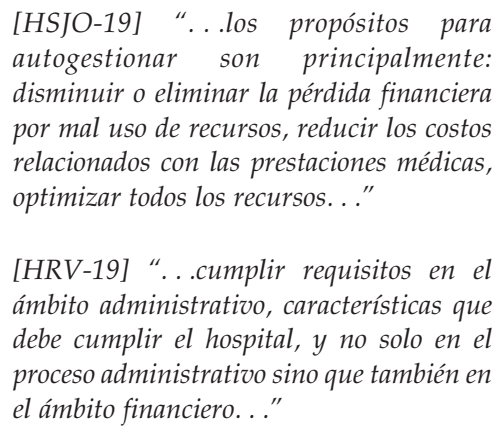

[HRV-19] “. . .cumplir requisitos en el ámbito administrativo, características que debe cumplir el hospital, y no solo en el proceso administrativo sino que también en el ámbito financiero. .."

De esta forma, el significado, el objetivo y el diseño de la política de autogestión hospitalaria se perciben desde la función de financiación de los sistemas de salud, esta última centrada principalmente en la contención y control de los costos en la gestión hospitalaria.

\section{Categoría de implementación de cambios organizacionales}

En las subcategorías de cambios y fortalezas organizacionales, la etapa de implementación de la política de autogestión no ha presentado mayores fortalezas para los tomadores de decisiones. No obstante, sí destacaron el alto nivel de compromiso de los equipos clínicos y administrativos en la transición al nuevo modelo organizacional. En el caso de la subcategoría de obstáculos organizacionales, la débil coordinación asistencial, la asimetría de información y la ausencia de competencias geren- ciales se identificaron como obstáculos para el desempeño eficiente de los hospitales.

\section{[HSJO-09] “. . . yo creo que la dificultad ha sido la coordinación, y lo otro implementar un sistema de registro, de costos, porque para ser autogestionados el hospital va a tener que generar sus recursos. .." \\ [HRV-06] ". . . a nivel organizacional, creo que [la dificultad ha sido] la poca difusión que se tiene hacia las bases, te llegan los documentos con un mes de atraso, cuando ya tienes que empezarlo a cumplir al día siguiente. .."}

Asimismo, en la misma subcategoría, los profesionales identificaron como elemento obstaculizador de la implementación la ausencia de políticas específicas sobre temas como competencias profesionales, disponibilidad de recursos humanos y estrategias de alineamiento de equipos profesionales para la ejecución de los cambios.

\section{[HSJO-08] “. . . principalmente la falta de profesionales que existe en el servicio y a nivel país, $y$ al tener unidades separadas $y$ centros de costos distintos, uno no puede compartir personal, porque tiene que estar diciendo yo te paso esto tú me pasas esto otro..." \\ [HRV-17] ". . .creo que es un poco eso, el cambio de paradigma que viene con los nuevos modelos de gestión que trajo la reforma. Esto último demandó más desafíos. Ahora, yo desconozco si la información habrá bajado a todos los niveles. .."}

En la subcategoría equipos de salud, los tomadores de decisiones identificaron en su desempeño un factor determinante para la implementación de los cambios organizacionales. Asimismo, reconocen la motivación y organización de los equipos como características determinantes que permitieron implementar los cambios en los hospitales.

[HSJO-10] ". . .creo que en ese sentido es lo mismo, que está bien motivado y conoce su trabajo ... digamos que todos tenemos la intención de hacer bien nuestro trabajo, más allá de las diferencias personales. .."

[HRV-12] "...lo que yo veo en este hospital es que tiene equipos estructurados que están trabajando en la autogestión, hay gente que está con designación de responsabilidades que tiene súper claros sus planes de trabajo. .."
Para los tomadores de decisiones, la etapa de implementación de la política de autogestión ha estado determinada por la ausencia de capacidades institucionales, situación que ha incidido negativamente en su percepción sobre el desempeño alcanzado por los hospitales estudiados en dicha etapa.

\section{Categoría de políticas y estrategias}

En las expectativas de los tomadores de decisiones, los ámbitos de los recursos humanos y la financiación de los hospitales constituyen áreas prioritarias para el diseño de nuevas políticas y estrategias que permitan avanzar hacia la implementación efectiva de la autogestión hospitalaria. En la subcategoría recursos humanos, los entrevistados consideraron que hacían falta nuevas competencias profesionales, administrativas y de provisión de servicios de salud, alineadas a las demandas de la política de autogestión.

\footnotetext{
[HSJO-02] ". . .lo primordial es generar un cambio de mentalidad en el recurso humano, para lograr alinear a todos tras un mismo objetivo. Se deben mejorar todos los procesos y capacitar a los funcionarios en este nuevo modelo de gestión. .."
[HRV-09] “...[lo] primero [es] educar a toda la gente que esté involucrada en el tema, desde la jefatura hasta el último funcionario ... dando a conocer, entusiasmando, encantando a la gente con el tema. Va a sonar a lo mejor duro, pero habría gente que cambiar. ."

En la subcategoría de financiamiento, para los tomadores de decisiones es perentorio mejorar el sistema de asignación de recursos de los hospitales con objeto de optimizar su eficiencia y autonomía presupuestaria.

[HSJO-11] ". . . creo que desde el punto de vista macro, como siempre [es necesario] mejorar la infraestructura y los recursos de dinero que deben fluir hacia los hospitales directamente y no a través de los servicios..."

[HRV-19] “. . .cómo se establecen los marcos presupuestarios hospitalarios y cómo se distribuye el presupuesto [...] ordenamiento también de la parte financiera que permita que los hospitales trabajen ordenadamente y con el adecuado presupuesto..." 
Para los tomadores de decisiones, resulta pertinente desarrollar nuevas opciones de política para mejorar la implementación de la autogestión hospitalaria y el desempeño global de las instituciones, las cuales no fueron incluidas en el diseño de la reforma de la salud y de la política.

\section{DISCUSIÓN}

La autonomía hospitalaria se ha definido como una política dirigida a la contención de costos y la eficiencia hospitalaria $(27,28)$. Los resultados obtenidos son consistentes en evidenciar cómo la autonomía en la asignación de recursos y la gestión financiera son elementos claves para conceptualizar la política de autogestión de los hospitales. Las expectativas de eficacia de los tomadores de decisiones se perciben a partir de la descentralización de la toma de decisiones basada en la eficiencia financiera y no así desde la implementación de redes asistenciales.

Más aun, en Chile, estudios recientes han destacado la relevancia que los directores de hospitales autogestionados le otorgan a los recursos y la ejecución de los presupuestos (29). En los hospitales objeto del presente estudio, la percepción de los tomadores de decisiones respecto de la implementación de la política de autogestión evidencia el impacto del enfoque racional del diseño de las políticas de salud en Chile. Esta aproximación arriba abajo se traduce en que los tomadores de decisiones identifiquen solo ámbitos financieros objetivos de control como determinantes para cumplir los objetivos de la política de EAR. Más aún, impide que perciban los reales grados de autonomía que la política de EAR introduce en los hospitales en un modelo de redes integradas de servicios de salud y GES.

En la ejecución de la política en los HRV y HSJO, las percepciones de incertidumbre organizacional y desinformación se han posicionado como obstaculizadores para la coordinación asistencial. Al respecto, otros estudios han destacado el impacto negativo de la desinformación en la coordinación asistencial (30). De hecho, la incertidumbre de los equipos y gestores acerca de la incidencia en los cambios en los hospitales ha sido descrita en algunas experiencias recientes de implementación de autonomía hospitalaria en América Latina $(31,32)$.
Un hallazgo relevante fue que los tomadores de decisiones percibieron la ausencia de una política de recursos humanos alineada con las exigencias organizacionales y clínicas de la implementación de la política. La falta de una política de recursos humanos fue advertida como una brecha determinante entre el diseño y la implementación de la reforma de la salud y de la política de autogestión hospitalaria (15, 18, 33-35). A siete años de la promulgación de la reforma, todavía no se han evidenciado progresos estructurales. Y lo que es más, diversos autores han planteado un desacople entre las políticas de recursos humanos en salud y los procesos de cambio, así como la escasa relevancia otorgada a esta relación por los diseñadores de las reformas (36-39). En el caso de Chile, esta realidad, sumada a la relevancia otorgada a la implementación de las GES y a la débil voluntad de los actores involucrados en la política de recursos humanos en salud, podrían explicar por qué los tomadores de decisiones no perciben avances respecto de la política que el propio proceso de acreditación de los EAR les exige implementar.

Si bien se han planteado marcos conceptuales sobre la importancia de las competencias de trabajo en equipos directivos que impulsan transformaciones para la gestión de los $\operatorname{EAR}(40,41)$, los resultados obtenidos evidencian el modo en que la ausencia de competencias organizacionales y gerenciales y de estrategias de capacitación para los equipos de salud ha impedido alcanzar los estándares que la autogestión demanda a los hospitales. Existe evidencia sobre la importancia de evaluar los procesos de gestión con el objetivo de mejorar la satisfacción de los usuarios en los hospitales públicos en Chile (42). Así, superar dichos obstáculos será determinante para mejorar la provisión de servicios de salud y satisfacer las expectativas de los usuarios.

La presencia de nudos críticos en la implementación de la política de EAR plantea la relevancia de alinear elementos del diseño de las políticas con las capacidades organizacionales de implementación. De modo similar a otros autores que identificaron la necesidad de generar capacidad de liderazgo para conducir eficazmente la implementación de los cambios (43), los resultados del presente estudio demuestran la relevancia de abordar el liderazgo de los tomadores de decisiones de los hospi- tales como un factor clave para dicha implementación.

Al propio tiempo, y aun cuando no fue una percepción manifestada por los entrevistados, un aspecto importante para desarrollar liderazgo y eficacia en la implementación de la política de autogestión es el diseño de estrategias de atracción y retención de directivos hospitalarios. Investigaciones han establecido cómo la falta de reconocimiento y compromiso, más que aspectos pecuniarios, se constituyen en factores desmotivadores para los directivos hospitalarios en Chile (44). Lo anterior podría ser explicado por los obstáculos de los directivos para promover cambios en instituciones complejas y altamente burocratizadas, características presentes en los sistemas de salud de América Latina. Este escenario destaca la importancia del reclutamiento de gestores profesionales, como un factor positivo para la calidad de los servicios públicos de salud (45).

Las limitaciones metodológicas que implican el diseño y la técnica de muestreo cualitativo utilizados en el presente trabajo determinan la necesidad de conducir nuevas investigaciones que exploren las dimensiones causales de las percepciones de los tomadores de decisiones de los hospitales aquí examinados, indagando también sobre la implementación de los requisitos solicitados a los hospitales para ser acreditados como EAR. Por ser un estudio descriptivo y basado en percepciones, estos aspectos no emergieron como temas clave pero sí fueron abordados implícitamente como parte de los obstáculos de la implementación.

Las percepciones de los tomadores de decisiones permiten confirmar la relevancia otorgada a la autonomía financiera como objetivo principal de la política de EAR, a la brecha existente entre los elementos del diseño y los de implementación de la política $\mathrm{y}$, aún más, a la necesidad de alinear el diseño a las capacidades de implementación de los equipos de salud. Para futuras experiencias de reforma en América Latina que involucren cambios a la política de hospitales, los datos probatorios aquí obtenidos tornan recomendable alinear las etapas de diseño e implementación de las políticas de salud y también asignar prioridad a la capacitación de los tomadores de decisiones y equipos de salud sobre los cambios a introducir. Finalmente, es también crítico ejecutar los cambios a través del diseño de políticas 
y estrategias que se acoplen a los nuevos modelos de gestión y atención que la política hospitalaria busca implementar.

Agradecimientos. Esta investigación fue patrocinada por la Dirección de In- vestigación y Desarrollo de la Universidad Austral de Chile (Código S-2010-37). Asimismo, utiliza fundamentos conceptuales y metodológicos provenientes del Proyecto FONDECYT No. 1101031 de la Comisión Nacional de Investiga- ción Científica y Tecnológica de Chile (CONICYT).

Conflicto de intereses. Ninguno declarado pos los autores

\section{REFERENCIAS}

1. Echeverri O. Mercantilización de los servicios de salud para el desarrollo: el caso de Colombia. Rev Panam Salud Publica. 2008;24(3):210-6.

2. Homedes N, Ugalde A. Las reformas de salud neoliberales en América Latina: una visión crítica a través de dos estudios de caso. Rev Panam Salud Publica. 2005;17(3):210-20.

3. Lloyd-Sherlock P. Health sector reform in Argentina: a cautionary tale. Soc Sci Med. 2005;60:1893-903.

4. Belmartino S. Una década de reforma de la atención médica en Argentina. Salud Colect. 2005;1(2):155-71.

5. García-Prado A, Chawla M. The impact of hospital management reforms on absenteeism in Costa Rica. Health Policy Plan. 2006;21(2):91-100.

6. Mesa-Lago C. Social security in Latin America: pension and health care reforms in the last quarter century. Lat Am Res Rev. 2007;42(2):181-201.

7. Figueras J, Musgrove P, Carrin G, Durán A. Retos para los sistemas sanitarios de latinoamérica: ¿qué puede aprenderse de la experiencia europea? Gac Sanit. 2002;16(1):5-17.

8. Hanson K, Atuyambe L, Kamwanga J, McPake B, Mungule O, Ssengooba F. Towards improving hospital performance in Uganda and Zambia: reflections and opportunities for autonomy. Health Policy. 2002;61:73-94.

9. Abdullah MT, Shaw J. A review of the experience of hospital autonomy in Pakistan. Int J Health Plann Manage. 2007;22(1):45-62.

10. Chile, Congreso de la Nación. Ley 19.937 de Autoridad Sanitaria y Gestión. Publicada en el Diario Oficinal el 24 de febrero de 2004.

11. Chile, Ministerio de Salud. Decreto No. 140 del Reglamento Orgánico de los Servicios de Salud. Publicado en el Diario Oficial el 21 de abril de 2005.

12. Nancuante U, Romero A. La reforma de la Salud. $1 .^{\mathrm{a}}$ ed. Santiago de Chile: Editorial Biblioteca Americana; 2008.

13. Retamal M. Autogestión hospitalaria en cifras. Rev Chil Salud Publica. 2009;13(3):169-74.

14. Artaza O, Méndez CA. Los establecimientos de autogestión en red. En: Grob C, ed. Gestión en salud: introducción a la administración de instituciones de salud. Valdivia: Editorial Universidad Austral de Chile; 2011. Pp. 72-9.

15. Sánchez S. Hospitales autogestionados en la red de salud de Chile: alcances y limitaciones de su ley. Cuad Med Soc. 2010;50(1):5-10.

16. Artaza O. Los desafíos de la autogestión hospitalaria. Rev Chil Pediatr. 2008;79:127-30.

17. Marshall C, Rossman GB. Designing qualitative research. 5. ${ }^{\text {a. }}$ ed. United States of America: Sage Publications; 2011.
18. Méndez CA, Alarcón A. Alineación del recurso humano y regulación de la competencia como factores claves para la autogestión hospitalaria en Chile. Rev Chil Salud Publica. 2011;15(2):90-7.

19. Onwuegbuzie AJ, Leech NL. A call for qualitative power analyses. Qual Quant. 2007;41: 105-21.

20. DiCicco-Bloom B, Crabtree BF. The qualitative research interview. Med Educ. 2006;40:314-21.

21. Kvale S. Las entrevistas en investigación cualitativa. Madrid: Morata; 2011.

22. Hsieh H, Shannon SE. Three approaches to qualitative content analysis. Qual Health Res. 2005;15(9):1277-88.

23. Elo S, Kyngäs $H$. The qualitative content analysis process. J Adv Nurs. 2008;62(1):107-15.

24. Onwegbuzie AJ, Leech NL. Validity and qualitative research: an oxymoron? Qual Quant. 2007;41:233-49.

25. Malterud K. Qualitative research: standards, challenges, and guidelines. Lancet. 2001;358:483-8.

26. Tobin GA, Begley CM. Methodological rigour within a qualitative framework. J Adv Nurs. 2004;48(4):388-96.

27. Unger JP, De Paepe P, Ghilbert P, Soors W, Green A. Disintegrated care: the Achilles heel of international health policies in low and middle-income countries. Int J Integr Care. 2006;6(18):1-13.

28. Sharma S, Hotchkiss D. Developing financial autonomy in public hospitals in India: Rajasthan's model. Health Policy. 2001;55: 1-18.

29. Cunill Grau N, Fernández MM, Vergara M. Gobernanza sistémica para un enfoque de derechos en salud: un análisis a partir del caso chileno. Salud Colect. 2011;7(1):21-33.

30. Henao Martínez D, Vázquez Navarrete ML, Vargas Lorenzo I. Factores que influyen en la coordinación entre niveles asistenciales según la opinión de directivos y profesionales sanitarios. Gac Sanit. 2009;23(4):280-6.

31. McPake B, Yepes F, Lake S, Sanchez L. Is the Colombian health system reform improving the performance of public hospitals in Bogotá? Health Policy Plan. 2003;18(2):182-94.

32. Machado AL, Giacone M, Alvarez C, Carri P. Health reform and its impact on healthcare workers: a case study of the National Clinical Hospital of Cordoba, Argentina. Soc Med. 2007;2(4):156-64.

33. Méndez CA. Los recursos humanos de salud en Chile: el desafío pendiente de la reforma. Rev Panam Salud Publica. 2009;26(3):276-80.

34. Méndez CA. Reflexión sobre la planificación de los recursos humanos y la autonomía de gestión en los hospitales de Chile. Rev Esp Salud Publica. 2009;83:371-8.

35. Méndez CA, Torres MC. Autonomía en la gestión hospitalaria en Chile: los desafíos para el recurso humano en salud. Rev Saude Publica. 2010;44(2):366-71.

36. Alwan A, Hornby P. The implications of health sector reform for human resources development. Bull World Health Organ. 2002;80:56-60.

37. Rigoli F, Dussault G. The interface between health sector reform and human resources in health. Human Resources for Health. Disponible en: http:/ / www.human-resourceshealth.com/content/1/1/9 Acceso el 01 de enero 2013.

38. Dussault G, Dubois CA. Human resources for health policies: a critical component in health policies. Human Resources for Health. Disponible en: http: / / www.humanresources-health.com/content/1/1/1 Acceso el 01 de enero de 2013.

39. Dussault G, Franceschini MC. Not enough there, too many here: understanding geographical imbalances in the distribution of the health workforce. Human Resources for Health. Disponible en: http://www. human-resources-health.com/content / 4/1/12 Acceso el 01 de enero de 2013.

40. Vergara M, Bisama L. Autogestión hospitalaria en red I: Competencias esenciales para la gestión. Rev Chil Salud Publica. 2009;13(3):163-8.

41. Vergara M, Bisama L. Autogestión hospitalaria en red II: El método. Rev Chil Salud Publica. 2010;14(1):52-8.

42. Riveros J, Berné C. Análisis de la opinión de usuarios sobre calidad percibida y satisfacción con hospitales públicos: estudio de caso desde la perspectiva de la aplicación del marketing. Rev Med Chil. 2007;135:862-70.

43. Sociedad Chilena de Administradores en Atención Médica y Hospitalaria. Los desafíos pendientes de la autogestión hospitalaria en red. Cuad Med Soc. 2009:49(3):171-7.

44. Bustamante-Ubilla MA, Del Río-Rivero MC, Lobos-Andrade GE, Villarreal-Navarrete PI. Percepción de la motivación de los directivos intermedios en tres hospitales de la Región del Maule, Chile. Salud Publica Mex. 2009;51:417-26.

45. Freire JM, Repullo JR. El buen gobierno de los servicios de salud de producción pública: ideas para avanzar. Cien Saude Colet. 2011;16(6):2733-42.

Manuscrito recibido el 27 de marzo de 2012. Aceptado para publicación, tras revisión, el 12 de octubre de 2012. 
ABSTRACT Objective. To learn the perceptions of decision-makers concerning the implementation stage of a hospital self-management policy in two highly complex hospitals in southern Chile.

Hospital self-management

Methods. A descriptive, exploratory, qualitative study based on semi-structured policy in Chile: perceptions in-depth interviews of decision-makers at the Regional Hospital of Valdivia and the Hospital San José de Osorno from August 2010 to December 2011. A convenience sample of 26 decision-makers was selected. The 26 interviews were recorded and transcribed verbatim. The information was analyzed using inductive content analysis. Results. The interviewees consider the concept of self-management to be determined by autonomy in decision-making about resource allocation and the financing of health service delivery in the hospitals. They also stated that human resources and financing policies should be included to improve the implementation stage. They related weaknesses with the lack of organizational capabilities and managerial skills in the health teams implementing the changes.

Conclusions. Conceptually, the hospital self-management policy is based on financial autonomy, and implementation is affected by persistent capacity gaps in policy design.

Key words Health policy; hospital administration; health services; health services research; Chile; Latin America. 